\title{
Determination of fetal heart rate reactivity from a single 20 -min window of non-stress testing in compromised fetuses ${ }^{\star, \star *}$
}

\author{
Young-Sun Park' ${ }^{1}$ Jeong-Kyu Hoh ${ }^{2}$, Seung- \\ Kwon Koh ${ }^{3}$, Nam-Su Kim ${ }^{4}$ and Moon-Il Park ${ }^{3, * * *}$ \\ ${ }^{1}$ Department of Mathematics, College of Natural \\ Sciences, Hanyang University, Seoul, Korea \\ 2 Department of Obstetrics and Gynecology, College of \\ Medicine, University of California, Irvine, CA, USA \\ ${ }^{3}$ Department of Obstetrics and Gynecology, Hanyang \\ University Hospital, Seoul, Korea \\ ${ }^{4}$ Department of Pediatric Cardiology, Hanyang \\ University Hospital, Seoul, Korea
}

\begin{abstract}
Aims: To shorten the analysis time needed for non-stress testing (NST) without decreasing efficacy in compromised fetuses.

Methods: We selected 80 cases with a 5-min Apgar score $<7$ as a study group and 259 cases with a 5-min Apgar score $\geq 9$ as a control group. We applied four different criteria (A, B, C, and D) to each study and control group for the first 20-min window of NST data to evaluate reactivity. Criteria $A, B$, and $C$ consisted of conventional reactivity criteria according to amplitude (15 or 10 beats per minute), duration (15 or $10 \mathrm{~s}$ ) and weeks of gestation $(\leq 31, \geq 32)$, and criteria D combined criteria C with approximate entropy (ApEn).

Results: The sensitivity of criteria D $(91.25 \%)$ was greater than the other three criteria $(P<0.0001)$. The specificities of criteria C (96.14\%) and D (99.23\%) were also higher than criteria $A$ and $B(P<0.0001)$. The positive and negative predictive value of criteria $D$ were better than that of criteria $C(97.33$ vs. $83.87, P=0.0066)$ and $(97.35$ vs. $89.89, P=0.0004)$, respectively.
\end{abstract}

\footnotetext{
*Condensation: The addition of approximate entropy to conventional criteria makes it possible to determine NST reactivity within a single 20 -min window.

**This work was supported by the Cluster Research Fund of Hanyang University (HY-2007-C).

${ }^{* \star *}$ Corresponding author:

Moon-II Park, MD, PhD

Department of Obstetrics and Gynecology

College of Medicine

Hanyang University

17 Haengdang-dong

Sungdong-gu

Seoul

Korea (133-792)

Tel.: + 82-2-2290-8409

Fax: +82-2-2297-8409

E-mail: parkmi@hanyang.ac.kr
}

Conclusion: Adding ApEn to the conventional criteria for reactivity shortened NST analysis time without decreasing efficacy, facilitating a decision of reactivity within a single 20-min NST window.

Keywords: Approximate entropy; non-stress testing; reactivity.

\section{Introduction}

Electronic fetal heart rate (FHR) monitoring is used for decades as an effective tool to evaluate fetal health. The non-stress testing (NST) is a useful application of electronic FHR monitoring for predicting antepartum fetal well-being in both high-risk and normal pregnancies. The NST was based on FHR response associated with fetal movements and was categorized as reactive or nonreactive [10]. The establishment of interpretation criteria has made the NST highly accurate for identifying healthy fetuses without restriction.

In 1997, the National Institute of Child Health and Human Development Research (NICHD) on Fetal Monitoring developed standardized and unambiguous definitions for fetal heart rate tracings [24]. FHR acceleration is defined as peaking at least 15 beats per minute (bpm) higher than the baseline and lasting $15 \mathrm{~s}$ from baseline to baseline. Before 32 weeks' gestation, accelerations usually peak at $10 \mathrm{bpm}$ and persist for $10 \mathrm{~s}[4,9,35]$. The results of an NST are considered reactive (reassuring), with a close relationship with fetal well-being, if two or more FHR accelerations are detected within a 20-min period, with or without fetal movement discernible by the mother [1, 26].

In contrast, a non-reactive tracing is one without sufficient FHR accelerations in a 40-min period [1, 26]. Such tests require a longer testing time and back-up tests such as acoustic stimulation test (AST), contraction stress test (CST), biophysical profile (BPP) or Doppler velocity for determining whether the fetus is in good health or not [32]. If a reactive result is not found after more than about 50 min of testing, the time to delivery must be decided promptly depending on the clinical scenario. Under emergency conditions, a test requiring more than $50 \mathrm{~min}$ could cause unfavorable or tragic fetal outcomes.

The purpose of this study was to find a method to shorten the necessary time for NST analysis without 
decreasing efficacy. NST data of depressed 5-min Apgar scores $(<7)$ cases with bad fetal outcomes were used to evaluate whether our proposed criteria could distinguish reactive from non-reactive cases within the first $20 \mathrm{~min}$ of NST recording.

\section{Materials and methods}

We performed a retrospective observational study using previously recorded data from the Hanyang Fetal Monitoring (HYFMII) system [27]. Among pregnant women who visited the outpatient obstetrics clinic for NSTs at Hanyang University Hospital from January 2005 to February $2008(n=1285), 80$ cases (6.23\%) with 5-min Apgar scores $<7$ were selected as a study group and 259 cases (20.16\%) with 5-min Apgar scores $\geq 9$ without adverse fetal outcomes or perinatal complications were randomly selected for the control group. The study group included preterm labor ( $<37$ weeks), small for gestational age (SGA) fetuses, preeclampsia, abruptio placentae, and fetal distress, among others (Table 2). Fetuses included in the study group had one or more adverse outcomes or perinatal complications. For example, in cases of abruptio placentae they also had other complications; preterm labor, preeclampsia, fetal distress and death. SGA case is not a complication that poses any urgent threat to the fetus but was included in the study group for qualifying a bad outcome with 5-min Apgar scores $<7$. This study did not require Institutional Review Board approval due to its retrospective approach in evaluating NST results.

All cases were single pregnancies with gestational ages between the 30 and 42 weeks, calculated from the last menstrual period. At the time of recording, pregnant women in labor or taking drugs that could affect FHR variability were excluded. All subjects were in a semirecumbent position for a minimum of $10 \mathrm{~min}$ before data collection, all of which occurred from 2 to $6 \mathrm{pm}$. FHR time series were recorded for $>40 \mathrm{~min}$ using the HYFM system, during which external fetal movements and breathing were included. The recorded data were sampled into a personal computer with a digital serial interface. Whenever missing data were found, they were recorded as zero. When the off-line FHR data of zero (missing data) or below $60 \mathrm{bpm}$ or above $200 \mathrm{bpm}$ were encountered, they were removed. From the heart rate data, corresponding R-R intervals were calculated. They were $1000-\mathrm{Hz}$ linearly interpolated by their $\mathrm{R}-\mathrm{R}$ interval to construct a real-time series of R-R intervals, and $2 \mathrm{~Hz}$ sub-sampled. We extracted and analyzed a $20-\min (\times 60 \times 2 \mathrm{~Hz}=2400$ points) time series of $R-R$ intervals during which fetal movements actively occurred. We finally analyzed 1000 points of time-series data extracted from a total of 2400 data points [19, 27]. We analyzed only the first 20 min of NST data to determine whether we could predict a bad prognosis within $20 \mathrm{~min}$. Results were regarded as non-reactive when reactive criteria were not met within $20 \mathrm{~min}$.

Four different criteria (A, B, C, and D) were applied to each group:

A. A reactive pattern defined as the presence of two or more accelerations lasting $\geq 15 \mathrm{~s}$ and reaching a peak of $\geq 15 \mathrm{bpm}$ above baseline within a 20 -min period.
B. A reactive pattern defined as the presence of two or more accelerations lasting $\geq 10 \mathrm{~s}$ and reaching a peak of $\geq 10 \mathrm{bpm}$ above baseline within a 20 -min period.

C. Criterion $A$ applied $\geq 32$ weeks of gestation, and Criterion B applied $<32$ weeks of gestation.

D. Criterion $\mathrm{C}$ with the addition of approximate entropy (ApEn) (cut-off value: 0.85 ).

ApEn, a measure of system complexity and irregularity was introduced by Pincus [28], and first applied to FHRs by Pincus and Viscarello [31]. ApEn is defined as the logarithmic likelihood that the patterns of data that are close to each other will remain close for the next comparison with a longer pattern. ApEn is computed using three parameters: the total number of data points $(N)$ in the R-R interval time series, the tolerance $(r)$, the embedding dimension $(m) . N$ was fixed at 1000 points, which corresponded to 7-8 min of NST time in our HYFM-II system [27], and $m$ at 2. The tolerance $r$ was chosen as $20 \%$ of the standard deviation (SD) of the R-R intervals and the correlation integral $C^{m}(r)$ was the number of vectors with a maximum distance $\leq r$ to the template vector. The natural logarithm of $C^{m}(r)$ was averaged over 1000 beats and this process was repeated for $m=2[19,31]$. Two input variables, $m$ and $r$, must be fixed to compute approximate entropy, and $m=2$ and $r=20 \%$ of the SD of the data sets were chosen as suitable and widely applied values on the basis of previous findings of good statistical validity $[15,19,22,28,29,31]$. We set the cut-off value for reactivity at 0.85 based on the results of a ROC analysis (Figure 1) [3, 12].

We used the concept of the diagnostic accuracy (sensitivity and specificity values) as decision-making tools with a receiver operator characteristics (ROC) analysis [3, 12]. To establish a cut-off value, ROC curve was created with approximate entropy

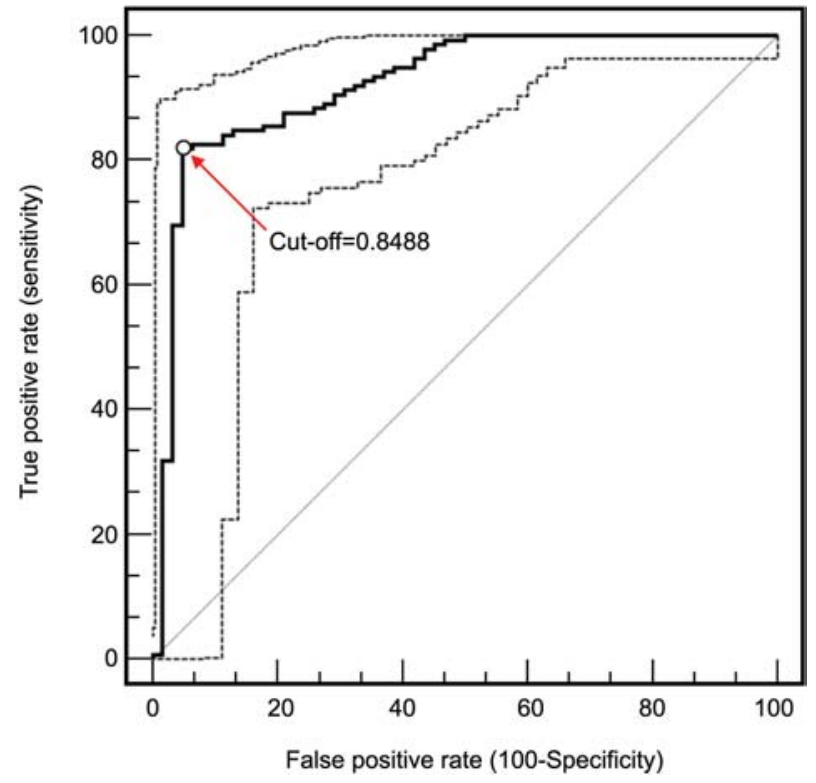

Figure 1 The reciprocal relationship between sensitivity and specificity by the receiver operator characteristics curve as a function of changing the decision criterion.

Area under the ROC curve $=0.92(\mathrm{SE}=0.0184)$, sensitivity $=$ $81.25 \%$, specificity $=94.21 \%$, Dot circle $(\bigcirc)$; cut-off $>0.8488$, dot line: $95 \%$ upper and lower confidence limit. 
as independent variable and low Apgar score as dependent variable. Later, sensitivity, specificity, positive predictive value, and negative predictive value were calculated for the cut-off value obtained. Intrinsic test performance is directly related to overlap between normal and abnormal populations. The ROC curve is a good way to demonstrate the relative performance of diagnostic tests: the superior test ROC curve can be seen to be closer to the upper left-hand corner of the graph.

Each variable was confirmed as not following a normal (Gaussian) distribution according to the Shapiro-Wilk test [36]. We used the Student's $t$-test to evaluate differences in FHR parameters (gestational age, mean baseline FHR, number of fetal movements, amplitude, mean minute range and approximate entropy) and neonatal outcomes (delivery weeks, baby height, birth weight, Apgar score and maternal age) between study and control groups. Categorical variables (gender) and predictive value for the four reactivity criteria were analyzed by Fisher's exact test and Pearson's $\chi^{2}$, respectively [41]. All analyses were performed using an alpha level of 0.05 as the threshold for statistical significance.

\section{Results}

Maternal age, gender, gestational age and mean baseline FHR were not statistically different between the study and control groups $(P>0.05)$. All other FHR parameters and neonatal outcomes were significantly different between groups $(P<0.01)$. The mean $A p E n$ was lower for the study $(0.69 \pm 0.16)$ compared to the control group (0.96 $\pm 0.08, \mathrm{P}<0.0001)$ (Table 1).

The study group included 61 patients with fetal distress; including 12 cases (19.67\%) born to mothers who had preeclampsia, and 9 (14.75\%) SGA infants (Table 2).
Figure 1 shows the ROC curve for ApEn using the cutoff values given in Table 3 (Criterion D). The test was considered appropriate, since the analyzed variable was a good predictor for a low Apgar score with an area below the diagonal of the curve significantly above $90 \%$. The best cut-off value for ApEn was about 0.85 , with $81.25 \%$ sensitivity and $94.21 \%$ specificity, suggesting that this a good parameter to predict low Apgar score (Figure 1).

The sensitivity of criterion D (91.25\%) was greater than that of the other three criteria, $(\mathrm{P}<0.0001)$. The specificities of criteria $C(96.14 \%)$ and D (99.23\%) were higher than criteria $A$ and $B(P<0.0001)$. The positive and negative predictive value of criterion $D$ were better than that of criterion $\mathrm{C}$ ( 97.33 vs. $83.87, \mathrm{P}=0.0066$ and 97.35 vs. 89.89, $\mathrm{P}=0.0004$, respectively), (Table 3 ).

\section{Discussion}

The assessment of FHR variability and its significance in predicting fetal well-being received increasing attention in recent years. Variations of the heart rate are attributed to modulation of the depolarization rate of the sinoatrial node through a number of physiological mechanisms such as blood pressure control, thermoregulation and respiration [6]. FHR is thought to reflect fetal well-being [21]. Computer analysis of cardiotocographs has the theoretical advantage of providing a reproducible and objective interpretation of FHR tracings by quantifying parameters that are difficult to assess by the human eye, such as short- and long-term variability. Several studies

Table 1 General characteristics of study and control groups.

\begin{tabular}{lllr}
\hline & $\begin{array}{l}\text { Study group* } \\
(\mathrm{n}=80)\end{array}$ & $\begin{array}{l}\text { Control group } \\
(\mathrm{n}=259)\end{array}$ & P-value \\
\hline Gestational age (weeks) & $38.36 \pm 1.76$ & $38.82 \pm 1.81$ & $\mathrm{NS}$ \\
Mean FHR (bpm) & $143.20 \pm 9.71$ & $143.36 \pm 8.61$ & $\mathrm{NS}$ \\
FM (No./20 min) & $1.94 \pm 2.59$ & $2.88 \pm 3.54$ & 0.0017 \\
AMP (bpm) & $15.26 \pm 5.34$ & $17.62 \pm 6.34$ & 0.0027 \\
MMR (ms) & $47.15 \pm 16.86$ & $53.76 \pm 19.17$ & 0.0022 \\
Acc 1515 & $1.19 \pm 2.31$ & $2.64 \pm 3.41$ & $<0.0001$ \\
Acc 1010 & $3.06 \pm 3.57$ & $5.08 \pm 4.74$ & $<0.0001$ \\
ApEn & $0.69 \pm 0.16$ & $0.96 \pm 0.08$ & $<0.0001$ \\
Delivery weeks (weeks) & $39.26 \pm 1.78$ & $40.00 \pm 1.33$ & 0.0009 \\
Gender (female) & $34(42.50)$ & $129(49.81)$ & $\mathrm{NS}$ \\
Baby height (cm) & $47.77 \pm 3.56$ & $49.75 \pm 2.08$ & 0.0017 \\
Baby weight (g) & $2,747 \pm 661$ & $3,267 \pm 340$ & $<0.0001$ \\
Apgar score 1 min & $5.24 \pm 2.09$ & $7.03 \pm 0.30$ & $<0.0001$ \\
Apgar score 5 min & $5.61 \pm 0.96$ & $9.00 \pm 0.06$ & $<0.0001$ \\
Maternal age (years) & $29.55 \pm 3.04$ & $29.41 \pm 3.47$ & $\mathrm{NS}$ \\
\hline
\end{tabular}

Data are represented as mean \pm standard deviation or frequency (\%).

$\mathrm{NS}=$ not significant, $\mathrm{P}>0.05$ by Student's $t$-test or Fisher's exact test.

${ }^{*}$ Apgar score 5 min $<7$; ${ }^{\dagger}$ Apgar score 5 min $\geq 9$.

$\mathrm{bpm}=$ beats per minute, mean $\mathrm{FHR}=$ mean baseline FHR (bpm), FM=number of fetal movements (No./20 min), AMP =amplitude $(\mathrm{bpm}), \mathrm{MMR}=$ mean minute range $(\mathrm{ms})$, Acc 1515 [1010] = number of accelerations for $15 \mathrm{bpm}-15 \mathrm{~s}$ [10 bpm-10 s], ApEn=approximate entropy. 
Table 2 Fetal outcomes and perinatal complications of the study group.*

\begin{tabular}{llcclrr}
\hline & $\begin{array}{l}\text { Preterm labor } \\
(<37 \text { weeks) }(\mathrm{n}=6)\end{array}$ & $\begin{array}{l}\text { SGA } \\
(\mathrm{n}=16)\end{array}$ & $\begin{array}{l}\text { Preeclampsia } \\
(\mathrm{n}=19)\end{array}$ & $\begin{array}{l}\text { Abruptio } \\
\text { placenta }(\mathrm{n}=4)\end{array}$ & $\begin{array}{l}\text { Fetal distress } \\
(\mathrm{n}=61)\end{array}$ & $\begin{array}{l}\text { Others } \\
(\mathrm{n}=6)^{\star \star}\end{array}$ \\
\hline Preterm labor $(<37$ weeks) & $6(100.00)$ & $1(6.25)$ & $5(26.32)$ & $1(25.00)$ & $4(6.56)$ & $2(33.33)$ \\
SGA & $1(16.67)$ & $16(100.00)$ & $5(26.32)$ & $2(50.00)$ & $9(14.75)$ & $2(33.33)$ \\
Preeclampsia & $5(83.33)$ & $5(31.25)$ & $19(100.00)$ & $2(50.00)$ & $12(19.67)$ & $1(16.67)$ \\
Abruptio placenta & $1(16.67)$ & $2(12.50)$ & $2(10.53)$ & $4(100.00)$ & $3(4.92)$ & $1(16.67)$ \\
Fetal distress & $4(66.67)$ & $9(56.25)$ & $12(63.16)$ & $3(75.00)$ & $61(100.00)$ & $2(33.33)$ \\
Others $^{\star *}$ & $2(33.33)$ & $2(12.50)$ & $1(5.26)$ & $1(25.00)$ & $2(3.28)$ & $6(100.00)$ \\
\hline
\end{tabular}

Data are represented as relative frequency (\%).

${ }^{*}$ Apgar score $5 \min <7(n=80)$; fetuses included in this study group had one or more bad fetal outcomes or perinatal complications. **Oligohydramnios or hydroamnios $(n=3,3.75 \%)$, CHVD $(2,2.50 \%)$, placenta previa $(1,1.25 \%)$.

$\mathrm{SGA}=$ small for gestational age, $\mathrm{CHVD}=$ chronic hypertensive vascular disease.

Table 3 Comparison of the reactivity according to four reactivity criteria (Criteria A, B, C and D) between study and control group.

\begin{tabular}{|c|c|c|c|c|c|}
\hline & & $\begin{array}{l}\text { Study group } \\
\mathrm{n}=80(\%)\end{array}$ & $\begin{array}{l}\text { Control group } \\
n=259(\%)\end{array}$ & $\begin{array}{l}\text { Positive } \\
\text { predictive } \\
\text { value (\%) }\end{array}$ & $\begin{array}{l}\text { Negative } \\
\text { predictive } \\
\text { value (\%) }\end{array}$ \\
\hline \multirow[t]{2}{*}{ Criterion A $(n=339)^{a}$} & Non-reactive & $47(58.75)$ & 49 (18.92) & 48.96 & 86.42 \\
\hline & Reactive & 33 (41.25) & 210 (81.08) & & \\
\hline \multirow[t]{2}{*}{ Criterion B $(n=339)^{b}$} & Non-reactive & $41(51.25)$ & 27 (10.42) & 60.29 & 85.61 \\
\hline & Reactive & 39 (48.75) & 232 (89.58) & & \\
\hline \multirow[t]{2}{*}{ Criterion C $(n=339)^{c}$} & Non-reactive & $52(65.00)$ & $10(3.86)$ & 83.87 & 89.89 \\
\hline & Reactive & $28(35.00)$ & $249(96.14)$ & & \\
\hline \multirow[t]{2}{*}{ Criterion D $(n=339)^{d}$} & Non-reactive & 73 (91.25) & $2(0.77)$ & 97.33 & 97.35 \\
\hline & Reactive & $7(8.75)$ & 257 (99.23) & & \\
\hline
\end{tabular}

Data are represented as frequency (\%).

${ }^{a}$ Criterion $\mathrm{A}$ : a reactive pattern defined as the presence of two or more accelerations lasting $\geq 15 \mathrm{~s}$ and reaching a peak of $\geq 15 \mathrm{bpm}$ above the baseline within a 20-min period, bCriterion B: a reactive pattern defined as the presence of two or more accelerations lasting $\geq 10 \mathrm{~s}$ and reaching a peak of $\geq 10 \mathrm{bpm}$ above the baseline within a 20-min period, ${ }^{\circ}$ Criterion C: Criterion $\mathrm{A}$ applied $\geq 32$ weeks of gestation, and Criterion B applied $<32$ weeks of gestation, ${ }^{d}$ Criterion D: Criterion $\mathrm{C}$ with the addition of approximate entropy (ApEn) (cut-off value: 0.85 ), ApEn=Approximate entropy, comparison of sensitivity, specificity, positive predictive and negative predictive value (Criteria $A-D) ; P<0.0001$, respectively.

$[11,17,38]$ have shown that computerized FHR analysis systems could minimize diagnostic errors and interpretative variability associated with the visual assessment of FHR patterns. Methods for FHR baseline and accelerations detection should be reproducible, as reproducibility is a major problem in FHR analysis [2]. Our data were already collected in HYFM system [27] which automatically saves all NST data. All data are retrievable at any time guaranteeing re-evaluation and reproducibility. Positive reports exist on the sensitivity and specificity of computerized analysis of antepartum tracings in detecting adverse outcomes [3]. However, the NST, which is a useful application of electronic FHR monitoring for predicting antepartum fetal well-being, has a high false-positive rate in the clinical setting [34]. Moreover, the positive predictive values of abnormal antenatal monitoring are more difficult to estimate, but are generally low [35].

ApEn has proven to be a powerful tool for the study of non-linearity in biological systems. For example, researchers have analyzed its ability to distinguish, over a short period, different physiological conditions in which the cardiovascular control system influences heart rate variability [16, 40]. Signorini et al. [39] proposed a new methodological approach for computerized cardiotocographic monitoring, based on multiparametric FHR analysis, that includes spectral parameters from autoregressive models and non-linear algorithms (i.e., ApEn). ApEn quantifies the irregularity in a time series by measuring randomness $[30,31]$. Consequently, a lower ApEn represents decreased randomness in heart rate, reflecting a lower complexity in the heart rate regulating system. Chaos theory suggests that systems with more regular dynamics are less resilient and cannot flexibly respond to or efficiently cope with various hemodynamic stresses [7, 8, 18, 25]. Some authors suggest that although ApEn is generally higher in reactive FHR patterns, it may be lower in the most reactive FHR patterns $[13,14,39]$ leading to errors in the interpretation of this difficult normal pattern [23]. However, it may be true that low ApEn can be detected in reactive FHR patterns, but it is also true that ApEn in most of problematic fetuses are low. Our study demonstrates the increased sensitivity by adding entropy to the already used linear criteria. In this study, we evaluated the detection of reactivity in the 
first 20-min window of NST data and found that addition of ApEn increased the sensitivity (validity or accuracy for reactivity) of determining reactivity to $91.25 \%$ compared with conventional visual interpretation criteria $(58.75 \%$, $51.25 \%, 65.00 \%$ for criteria A, B, and C, respectively). The cut-off value of ApEn to discriminate between reactive or non-reactive results was 0.85 in this study. We previously measured the mean value of ApEn in SGA cases as $0.59-0.71$ [19]. In contrast, Pincus and Viscarello suggest that a value of $\mathrm{ApEn}$ below 0.80 can predict high-risk conditions such as asphyxia [31]. When we applied cut-off values of 0.85 the apparent error rate was $2.65 \%$ (false positive cases $=7$; false negative cases $=2$ ).

In general, the NST can be continued for an additional $40 \mathrm{~min}$ or longer if conclusive results are not observed [1]. If conclusive results are still not available after more than 40-50 min, or if the case requires expedited decision-making, our method offers a reasonable opportunity to decrease the necessary interpretation time for the NST.

It is important to keep in mind that clinical evaluations performed on shorter time intervals may be misleading [33]. Even so, our method could be useful in urgent conditions that need immediate results to guide treatment. Furthermore, because the reactive criteria together with ApEn are sensitive enough to catch minimal signals that may be overlooked with visual interpretation, they may help detect minor abnormalities of the cardiovascular control system [5, 20, 31, 37].

The adoption of ApEn for reactive NST tracing has diagnostic value for predicting abnormal conditions of the fetal cardiovascular system, including abnormal minimal heart behavior that might be concealed by linear interpretation methods. The standard analysis of FHR variability by conventional criteria defines acceleration, deceleration and baseline variability, but lacks the robustness needed to identify and quantify pattern regularity. The addition of ApEn to the criteria for NST reactivity may help overcome such limitations of existing problems. In conclusion, adding ApEn to the conventional criteria for reactivity shortens NST analysis time without decreasing efficacy, facilitating a decision about reactivity within a single 20-min NST window in compromised fetuses.

\section{References}

[1] American Academy of Pediatrics and American College of Obstetricians and Gynecologists. Guidelines for perinatal care, sixth ed. Washington, DC: American Academy of Pediatrics; 2008.

[2] Ayres-de-Campos D, Bernardes J, Costa-Pereira A, Pereira-Leite L. Inconsistencies in expert's classification of cardiotocograms and subsequent clinical decision. $\mathrm{Br} \mathrm{J}$ Obstet Gynaecol. 1999;106:1307-10.

[3] Ayres-de-Campos D, Costa-Santos C, Bernardes J, SisPorto Multicentre Validation Study Group. Prediction of neonatal state by computer analysis of fetal heart rate tracings: the antepartum arm of the SisPorto multicentre validation study. Eur J Obstet Gynecol Reprod Biol. 2005; 118:52-60.

[4] Brown R, Patrick J. The nonstress test: How long is enough? Am J Obstet Gynecol. 1981;141:646-51.

[5] Chatterjee S, Yilmaz MR, Habibullah M, Laudato M. An approximate entropy test for randomness. Communications in statistics. Theory and Methods. 2000;29:655-75.

[6] Dalton KJ, Dawes GS, Patrick JE. The autonomic nervous system and fetal heart rate variability. Am J Obstet Gynecol. 1983;146:456-62.

[7] di Renzo GC, Branconi F, Clerici G, Fioriti V, Montani M, Pardini A, et al. Fractal analysis: a new method for evaluating fetal heart rate variability. J Perinat Med. 1996;24: 261-9.

[8] Elbert T, Birbaumer N, Graf KE, Kowalik ZJ, Ray WJ, Skinner JE. Chaos and physiology: deterministic chaos in excitable cell assemblies. Physiol Rev. 1994;74:1-47.

[9] Electronic fetal heart rate monitoring: research guidelines for interpretation. National institute of child health and human development research planning workshop. Am J Obstet Gynecol. 1997;177:1385-90.

[10] Evertson LR, Gauthier RJ, Schifrin BS, Paul RH. Antepartum fetal heart rate testing. I. Evolution of the nonstress test. Am J Obstet Gynecol. 1979;133:29-33.

[11] Gagnon R, Campbell MK, Hunse C. A comparison of visual and computer analysis of antepartum fetal heart rate tracings. Am J Obstet Gynecol. 1993;168:842-7.

[12] Garcia GS, Mariani Neto C, Araujo Júnior E, Garcia RL, Nardozza LM, Moron AF. Fetal acidemia prediction through short-term variation assessed by antepartum computerized cardiotocography in pregnant women with hypertension syndrome. Arch Gynecol Obstet. 2008;278:125-8.

[13] Gonçalves H, Bernardes J, Rocha AP, Ayres-de-Campos $D$. Linear and nonlinear analysis of heart rate patterns associated with fetal behavioral states in the antepartum period. Early Hum Dev. 2007;83:585-91.

[14] Gonçalves H, Rocha AP, Ayres-de-Campos D, Bernardes J. Frequency domain and entropy analysis of fetal heart rate: appealing tools for fetal surveillance and pharmacodynamic assessment of drugs. Cardiovasc Hematol Disord Drug Targets. 2008;8:91-8.

[15] Gonçalves H, Rocha AP, Ayres-de-Campos D, Bernardes J. Internal versus external intrapartum foetal heart rate monitoring: the effect on linear and nonlinear parameters. Physiol Meas. 2006;27:307-19.

[16] Gonçalves H, Rocha AP, Ayres-de-Campos D, Bernardes $\mathrm{J}$. Linear and nonlinear fetal heart rate analysis of normal and acidemic fetuses in the minutes preceding delivery. Med Biol Eng Comput. 2006;44:847-55.

[17] Hiett AK, Devoe LD, Youssef A, Gardner P, Black M. A comparison of visual and automated methods of analyzing fetal heart rate tests. Am J Obstet Gynecol. 1993;168: 1517-21.

[18] Hogue CW Jr, Despotis GD, Domitrovich PP, Re L, Schuessler RB, Stein PK, et al. RR interval dynamics before atrial fibrillation in patients after coronary artery bypass graft surgery. Circulation. 1998;98:429-34.

[19] Hoh JK, Park YS, Cha KJ, Oh JE, Lee HJ, Lee GT, et al. Chaotic indices and canonical ensemble of heart rate patterns in small-for-gestational age fetuses. J Perinat Med. 2007;35:210-6. 
[20] Kaplan DT, Furman MI, Pincus SM, Ryan SM, Lipsitz LA, Goldberger AL. Aging and the complexity of cardiovascular dynamics. Biophys J. 1991;59:945-9.

[21] Kikuchi A, Hayashi A, Horikoshi T, Kozuma S, Shimizu T, Taketani $Y$, et al. Nonlinear analyses of heart rate variability in normal and growth-restricted fetuses. Early Hum Dev. 2006;82:217-26.

[22] Li X, Zheng D, Zhou S, Tang D, Wang C, Wu G. Approximate entropy of fetal heart rate variability as a predictor of fetal distress in women at term pregnancy. Acta Obstet Gynecol Scand. 2005;84:837-43.

[23] Matias A, Xavier P, Bernardes J, Patrício B. Fetal heartrate monitoring during maternal hypoglycaemic coma: a case report. Eur J Obstet Gynecol Reprod Biol. 1998;79: 223-5.

[24] National Institute of Child Health and Human Development Research Planning Workshop. Electronic fetal heart rate monitoring: research guidelines for interpretation. Am J Obstet Gynecol. 1997;177:1385-90.

[25] Nelson JC, Griffin MP, Moorman JR, Rizwan-uddin. Probing the order within neonatal heart rate variability. Pediatr Res. 1998;43:823-31.

[26] Pardey J, Moulden M, Redman CW. A computer system for the numerical analysis of nonstress tests. Am J Obstet Gynecol. 2002;186:1095-103.

[27] Park MI, Cha KJ, Hwang JH, Koh SK, Park YS. Computerized analysis of fetal heart rate parameters by gestational age. Int J Gynaecol Obstet. 2001;74:157-64.

[28] Pincus SM. Approximate entropy as a measure of system complexity. Proc Natl Acad Sci USA. 1991;88:2297-301.

[29] Pincus SM, Goldberger AL. Physiological time-series analysis: what does regularity quantify? Am J Physiol. 1994;266:H1643-56.

[30] Pincus SM, Singer BH. Randomness and degrees of irregularity. Proc Natl Acad Sci USA. 1996;93:2083-8.

[31] Pincus SM, Viscarello RR. Approximate entropy: a regularity measure for fetal heart rate analysis. Obstet Gynecol. 1992;79:249-55.
[32] Piyamongkol W, Trungtawatchai S, Chanprapaph P, Tongsong $\mathrm{T}$. Comparison of the manual stimulation test and the nonstress test: a randomized controlled trial. J Med Assoc Thai. 2006;89:1999-2002.

[33] Romanini C, Rizzo G. Fetal behaviour in normal and compromised fetuses. An overview. Early Hum Dev. 1995;43: 117-31.

[34] Sameshima H, Ikenoue T. Predictive value of late decelerations for fetal acidemia in unselective low-risk pregnancies. Am J Perinatol. 2005;22:19-23.

[35] Saraçoğlu F, Göl K, Sahin I, Türkkani B, Oztopçu C. The predictive value of fetal acoustic stimulation. $\mathrm{J}$ Perinatol. 1999;19:103-5.

[36] SAS Institute. SAS/QC Software: Usage and Reference I and II. Cary, NC: SAS Institute; 1995.

[37] Schifin BS, Clement D. Routine antepartum fetal heart rate monitoring. In: Fetal monitoring, physiology and techniques of antenatal and intrapartum assessment. Edit by Spencer JAD, Oxford University Press, 1991.

[38] Schneider E, Schulman H, Farmakides G, Paksima S. Comparison of the interpretation of antepartum fetal heart rate tracings between a computer program and experts. $J$ Matern Fetal Invest. 1991;1:205-8.

[39] Signorini MG, Arduni D, Cerutti S, Magenes G. Linear and nonlinear parameters for the analysis of fetal heart rate signal from cardiotocographic recordings. IEEE Trans Biomed Eng. 2003;50:365-74.

[40] Signorini MG, Cerutti S, Lombardi F, Sassi R. Regularity patterns in heart rate variability signal: the approximate entropy approach. Med Biol Soci. 1998;20:306-9.

[41] Stokes ME, Davis CS, Koch GG. Categorical data analysis using the SAS system, 2nd ed. SAS Institute Inc., Cary, NC; 2000.

The authors stated that there are no conflicts of interest regarding the publication of this article.

Received August 8, 2008. Revised September 24, 2008. Accepted January 26, 2009. Previously published online March 17, 2009. 